\title{
Administración municipal y políticas públicas para la creación de infraestructura en Cozumel, Quintana Roo
}

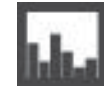 \\ REsUMEN \\ La infraestructura facilita la accesibilidad y permite a la \\ demanda turística trasladarse hacia un destino. Por ello, el \\ impulso al sector turístico requiere del soporte y asistencia \\ del sector público, teniendo en cuenta que el turismo trae \\ amplias y controversiales repercusiones, ligado a la \\ exigencia de más bienes públicos como parte del entorno \\ social en el que se habita o convive. Esta razón demanda a \\ las autoridades el manejo eficiente de recursos y la \\ implementación de políticas que coadyuven a la generación \\ de esa infraestructura, con el propósito de otorgar \\ beneficios a la sociedad. En este trabajo se evalúa la \\ administración municipal y las políticas públicas en el \\ municipio de Cozumel, durante los periodos 1996-1998 \\ y 1999-2000, identificando la eficiencia con la que se \\ captaron los ingresos y condujeron los gastos, haciendo \\ énfasis en la generación de obras públicas e infraestructura, \\ así como en las políticas públicas.

\footnotetext{
PALABRAS Cozumel, administración municipal, infraestructura, CLAVE políticas públicas.
}

Lucinda Arroyo Arcos

*Universidad de Quintana Roo / larroyo@correo.uqroo.mx 
Administración municipal y

políticas públicas para la

creación de infraestructura

\section{Introducción}

El presente trabajo tiene como objetivo evaluar la administración municipal en el manejo de los recursos destinados para obras públicas e infraestructura, así como las políticas públicas implementadas en el municipio con la misma intención. Aunque la captación de recursos (Hacienda municipal) no es el tema central de la investigación es necesario presentar su comportamiento y tendencias desde 1996 al 2000.

Para evaluar los resultados de la acción administrativa existen métodos muy diversos los cuales permiten apreciar los efectos políticos, económicos y sociales generados por la intervención gubernamental, en el caso particular se elige el enfoque clínico, partiendo de los objetivos establecidos en los planes de trabajo, verificando los resultados en los periodos establecidos, y señalando algunas consecuencias inesperadas (Guerrero citado en Aguilar, 1992).

De ahí que los criterios utilizados para identificar los resultados de la administración e implementación de políticas son: a) eficiencia, que significa lograr los objetivos con el menor de los costos; b) eficacia, que es la capacidad de hacer concreta o reales las metas programadas (logro de objetivos tangibles); c) igualdad, que implica el mismo tratamiento a todos los sectores sociales; d) equidad, que se preocupa en particular por las situaciones de desigualdad social, es decir, que a todos los ciudadanos correspondan los mismos derechos y mismo trato; d) pertinencia o adecuación, se refiere a la capacidad de responder satisfactoriamente a las necesidades y demandas sociales (ibidem, p. 6I). Los criterios antes mencionados sirven de parámetros en la evaluación realizada, sobre todo el apartado de gastos con énfasis en el rubro de infraestructura durante 1996-2000. Por otra parte, se identifican las políticas públicas que han coadyuvado a la generación de infraestructura, resaltando las fortalezas, debilidades, problemáticas y potencialidades de las mismas.

Finalmente, el análisis de datos y presentación de resultados se proponen como una herramienta para la toma de decisiones de los servidores públicos, para el avance en los procesos y uso de los recursos para beneficio de la sociedad. 


\section{Metodología}

Se revisaron trabajos relacionados principalmente con publicaciones sobre el municipio, retomando algunas de las técnicas aplicadas en ese tipo de estudios. Asimismo, se consultaron documentos oficiales estatales y municipales tales como: las actas de cabildo, los informes anuales de los presidentes municipales, el dictamen de la cuenta pública municipal 1996-2000, el Plan de Desarrollo Estatal, Plan de Desarrollo Municipal, Plan Subregional de Desarrollo, páginas del gobierno de Quintana Roo, entre otros. Se analizó la información y se detallaron los montos de ingresos y egresos del municipio, destinados a obras de infraestructura durante los periodos que considera la investigación.

Para la evaluación, tanto de la administración como de las políticas, se utilizó el método clínico, siendo este el más pertinente, pues considera los objetivos y el logro de los mismos, justificando hasta donde sea posible, por qué algunos no se concretaron, sin dejar al margen los beneficios alcanzados. A partir del enfoque establecido, se utilizan los criterios de eficiencia, eficacia, equidad y pertinencia para evaluar la generación de obras públicas e infraestructura y manejo de recursos, para ello se definieron parámetros de comparación; en primer lugar, la existencia de un plan de trabajo con objetivos claros, medibles y cuantificables; en segundo, el número de obras realizadas en los periodos de evaluación; en tercero, el número de colonias beneficiadas y por último, la cantidad de recursos erogados y los devueltos a la federación. En ese contexto, se procede a la evaluación de dos periodos administrativos 1996-1998 y 1999-2000.

Para reconocer y analizar las políticas implementadas en el municipio, se consultaron documentos oficiales: el Manual del Ramo 33, informes técnicos de la Dirección de Obras Públicas, y como complemento, se llevaron a cabo entrevistas en profundidad a informantes clave, del sector público (director de Obras Públicas, secretario General, subsecretario, ex tesorero municipal, ex director de Obras Públicas) y privado.Asimismo, se entrevistó al director de Administración Portuaria Integral (API), a los gerentes de los muelles turísticos Puerta Maya, Muelle Internacional, Punta Langosta y al gerente del Aeropuerto Internacional. Finalmente, se realizan las observaciones y conclusiones. 
Administración municipal y

políticas públicas para la

creación de infraestructura

\section{Análisis y discusión de resultados}

La Hacienda municipal está integrada por los bienes muebles e inmuebles y los recursos ordinarios: impuesto, productos (arrendamientos), derechos (servicios prestados), aprovechamientos (rezagos y multas), y los extraordinarios o eventuales como: las participaciones (recursos que se establecen a favor de los municipios en el convenio de coordinación en materia de administración de ingresos federales).

Sin embargo, en los municipios mexicanos existe una problemática para la captación de los recursos, el caso de Cozumel no es la excepción, y de acuerdo con el análisis de datos se detectó que en el periodo 1996-2000 existió una clara disminución, destacándose que los impuestos en 1997 fueron de $25 \%$, mismos que no se recuperaron en los dos años siguientes, por el contrario, bajaron hasta 14\% en 1999 y para el 2000 tan sólo se obtuvo $18 \%$ con respecto al total de los ingresos, esta baja captación se atribuye a moras en el pago de impuesto predial, descuentos y excepción del pago a particulares (Actas de cabildo, 1999). El rubro de productos presenta una disminución que va de $6 \%$ en 1996 a $3 \%$ en el año 2000 , a causa de la baja captación de multas, arrendamientos y rezagos. Por derechos, se observa que los ingresos tuvieron un comportamiento similar durante el periodo 1996-2000, entre 10 y $12 \%$; por aprovechamientos se observó una constante de $8 \%$ en 1997-1999 y una disminución a 5\% en el 2000; el concepto de otros ingresos presenta una pequeña disminución en los últimos tres años que van de $12 \%$ en 1998 a $9 \%$ en el 2000; los ingresos por participaciones presenta altibajos en los ingresos, de $49 \%$ en 1996 baja $40 \%$ en 1998 y se recupera en el 2000 hasta $46 \%$ (gráfica I).

En la gráfica 2, de acuerdo con el análisis realizado, se observa que los gastos en el municipio de Cozumel entre 1996-2000, se aplican en primer lugar a los servicios personales (remuneraciones), representando 44, 4I, 32, 35 y $38 \%$ respectivamente, aunque existe una disminución en los tres últimos años, siguió ocupando la primera posición; en segundo término se encuentran los gastos en servicios generales (todo tipo de servicios y contratación a particulares), con un promedio de 19.2\% durante el periodo; el tercer lugar corresponde a los egresos en obras públicas, donde se observa un incremento de $7 \%$ en 1997 a $19 \%$ en el 2000 , como resultado de la implementación de la política social a partir de 1998; en cuarto lugar, los gastos en materiales 
Gráfica I. Comportamiento de la hacienda municipal 1996-2000.

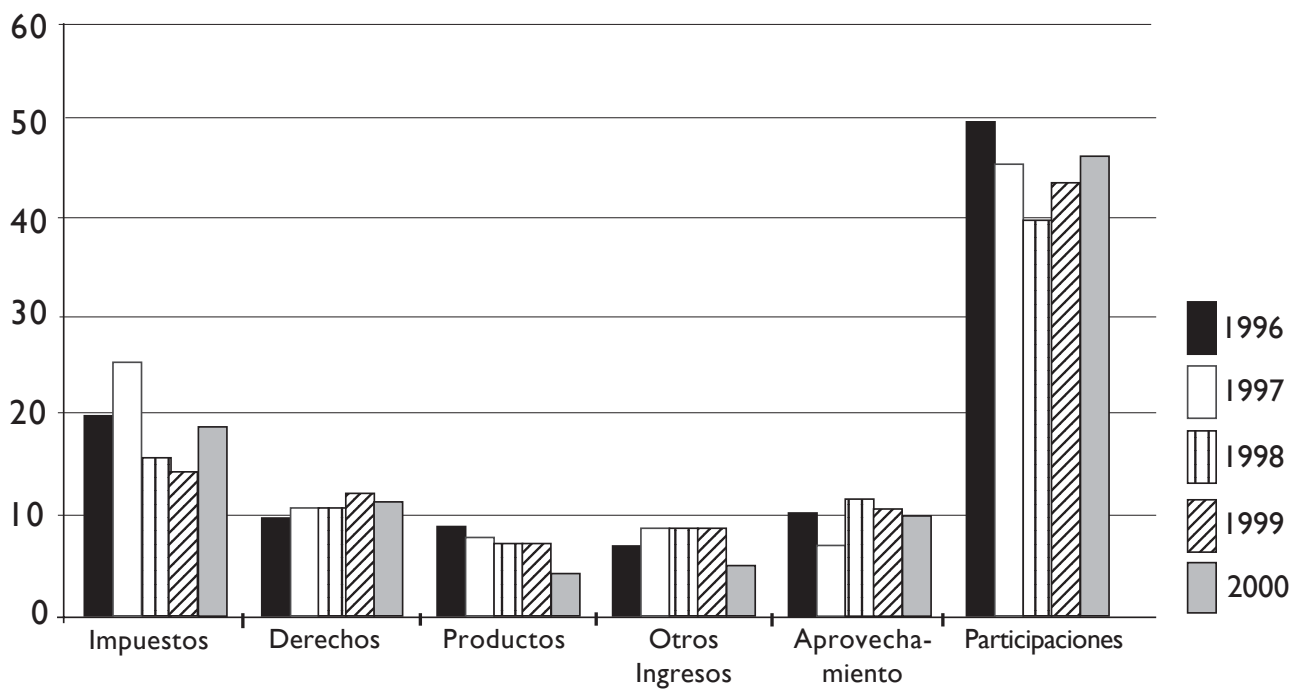

129 .

Fuente: Elaboración propia con base en el Dictamen Cuenta Pública del Municipio de Cozumel, ejercicio fiscal 1996-2000.

Gráfica 2. Comportamiento de los gastos municipales 1996-2000.

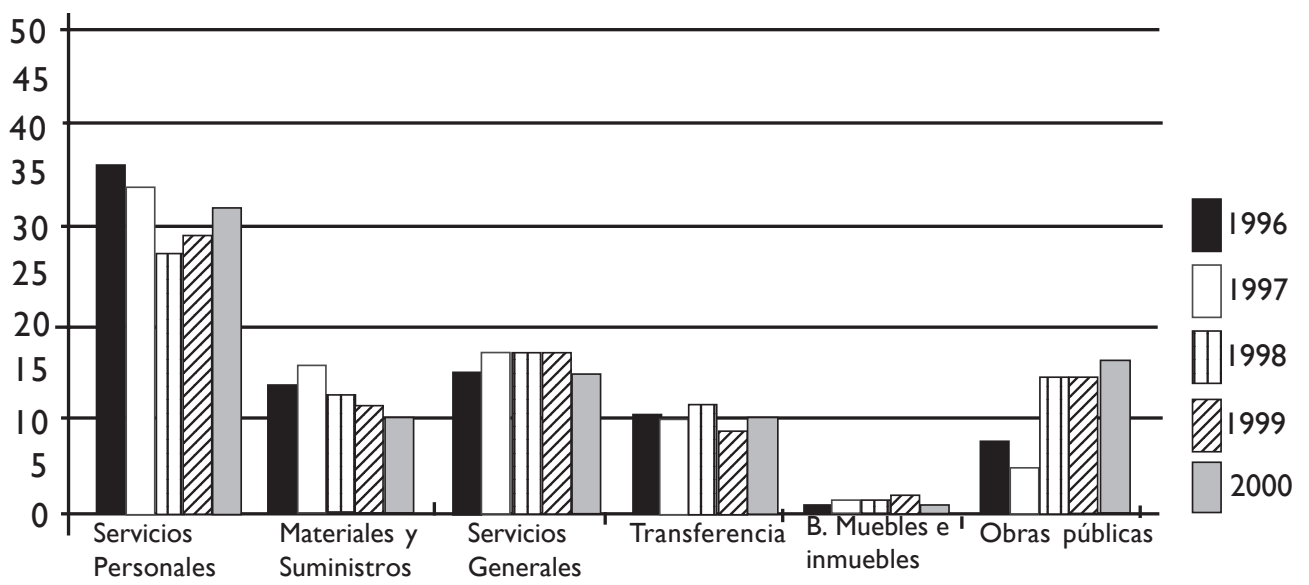

Fuente: Elaboración propia con base en el Dictamen Cuenta Pública del Municipio de Cozumel, ejercicio fiscal 1996-2000. 
Administración municipal y

políticas públicas para la

creación de infraestructura

y suministros (adquisición de toda clase de insumos) con una tendencia hacia abajo como se observa en 1997, de $18 \%$ y en el 2000 tan sólo de $12 \%$; el penúltimo lugar es ocupado por las transferencias (apoyos financieros o en especie) con un promedio de $12.2 \%$ en el periodo considerado, $y$ en último lugar, los egresos en bienes muebles e inmuebles representando en promedio I.8\% respecto de los gastos totales (gráfica 2 ).

Como resultado del análisis de la hacienda municipal y gastos públicos, tenemos que los ingresos propios presentaron una disminución durante 1996-2000, de 5I, 55, 50, 48 y 45\%, respectivamente. Esto significa que la captación de recursos cada vez es más difícil en el municipio de Cozumel y que depende en gran medida de las participaciones otorgadas por el gobierno federal y estatal. Por lo mismo, se refleja un déficit en 1996, 1998 y 1999 y tan sólo un superávit en 1997 y 2000 (Cuenta Pública Municipal, 1996-2000).

\section{Evaluación de la administración respecto a la generación de obras e infraestructura 1996-1998 y 1999-2000}

La evaluación se realizó tomando como parámetros de referencia los objetivos planteados en los planes municipales, para efectos de la investigación se definieron los periodos 1996-1998 y $1999-2000$ considerando la información vigente $y$ años cerrados del I de enero al 3I de diciembre. En el primer periodo oficial, la presidencia municipal estuvo dirigida por el Sr.Víctor Vivas González, su plan de trabajo se caracteriza por tener una presentación clara de los objetivos y metas a lograr al término de su mandato, presentando un diagnóstico de la situación presente, y proponiendo mejoras para atender las demandas sociales, esto facilita la evaluación del rubro de interés con los criterios de eficiencia, eficacia, equidad y pertinencia.

Durante la administración de 1996-1998, se realizaron 55 tipos de obras públicas e infraestructura incluyendo equipamientos y servicios, mismos que se encuentran registrados como obras en los documentos oficiales consultados, se beneficiaron a II colonias del municipio cuyas demandas coinciden con el tipo de obra otorgada. Los recursos utilizados durante los dos primeros años son municipales, y en el último año se incrementan por la implementación de la política social donde se etiquetan recursos del Ramo 33, para fomento de obras 
públicas y fortalecimiento municipal. Por lo tanto, de acuerdo con los resultados antes mencionados y con los criterios propuestos se entiende que la administración fue eficiente en obras e infraestructura, al llevar a cabo diversas acciones y satisfacer las demandas requeridas por la sociedad con menos recursos de los asignados y en el tiempo establecido; dejando en claro que el cumplimiento de objetivos incluidos en el plan de trabajo se lograron en $70 \%$. Sin embargo, se reconoce que se destinaron recursos a otro tipo de equipamiento o servicios no considerados ni planeados al inicio de la administración pero, a pesar de los esfuerzos realizados, no se logra la eficacia.

Para el segundo periodo, 1999-2000, la presidencia municipal estuvo dirigida por el Lic. Félix.A. González Canto, quien en su plan de trabajo no incluye un diagnóstico de la situación presente y futura, además los objetivos y metas planteadas son generales. Lo anterior complica la evaluación, sin embargo, vale la pena destacar que en ese periodo se realizaron un total de 83 obras, beneficiando a 14 colonias populares. Pero ante la falta de parámetros es imposible realizar la evaluación con los criterios de eficiencia y eficacia. Con respecto a la equidad, en ambos periodos municipales se subraya que la administración toma en consideración aquellas colonias que presentan características de rezago social y altos índices de marginalidad, en las que se desarrollaron obras e infraestructura, permitiéndoles el acceso a los servicios y respetándose la búsqueda de igualdad de beneficios en la comunidad; por lo tanto, este criterio se cumple satisfactoriamente.

Asimismo, los programas municipales implementados se consideran pertinentes; éstos se han enfocado al cumplimiento de requerimientos de obras e infraestructura en aquellas colonias de nueva creación y en las establecidas, lo que ha permitido disminuir la demanda de este tipo de solicitudes. En este sentido, se han aprovechado los recursos etiquetados para el fortalecimiento municipal, renovando al mismo tiempo la imagen urbana del municipio, principalmente en lo que se conoce como primer cuadro de la ciudad. Es importante señalar que los recursos asignados para la implementación de los programas municipales, no fueron erogados en su totalidad ya que entre 1998 y 2000 la ejecución fue de 78, 56 y 46\% respectivamente, en promedio el uso de los recursos fue de $60 \%$ en los últimos tres años del periodo considerado. Esto es el reflejo de una falta de 
Administración municipal y

políticas públicas para la

creación de infraestructura

planeación de actividades, lo que impide un mejor desempeño de las administraciones municipales.

\section{Políticas implementadas}

Una de las políticas para generar obras públicas e infraestructura es la política social que surge en 1998 como parte de los programas federales y que en coordinación con el gobierno del Estado de Quintana Roo se implementa en todos los municipios, teniendo como objetivo fundamental beneficiar a la población en condiciones de rezago social y de pobreza extrema, mediante el Fondo de Aportación Federal (FAF), apoyada por los programas con recursos del Ramo 33 como el Fondo para la Infraestructura Social Municipal (FISM), y el Fondo de Aportaciones para el Fortalecimiento Municipal (FAFM), (Manual de Ramo 33, 2003). Para el caso de los programas FISM y FAFM con recursos del Ramo 33, durante 1998 y 2000 se ejecutaron en obras e infraestructura 89,66 y $59 \%$ de los recursos, es decir, un promedio de $71 \%$. Retomando la parte de los recursos propios ejecutados y de los fondos etiquetados (FISM y FAFM) tenemos que solamente se erogaron $65 \%$ de los recursos asignados, lo que indica que $35 \%$ no se aplicó, mismos que se reintegraron a la federación. Esto es un claro ejemplo de la falta de un plan a largo plazo que permita ir cumpliendo con los objetivos establecidos y otorgar a la ciudadanía mejores servicios y obras públicas. En el cuadro I, se anotan las fortalezas, debilidades, amenazas y oportunidades de la Política social.

En el mismo contexto, otra de las políticas implementadas en todo el país a partir del modelo neoliberal, es la privatización de empresas en sus diversas modalidades, buscando la eficiencia de los servicios en manos de la iniciativa privada, mediante la transferencia total o parcial de los activos, aumento de capital mediante aportaciones inversionistas, venta de los activos con motivo de su disolución, celebración de contratos de arrendamiento, concesiones y otros similares (CEPAL, 2002)

Cabe señalar, que el gobierno federal desde 1996 ha otorgado concesiones en el municipio de Cozumel para la operación y manejo de servicios mediante la construcción de infraestructura, destacándose la concesión del Aeropuerto Internacional otorgada en 1998 a la compañía Aeropuertos del Sureste (ASUR) por un plazo de 50 años, presentando constantes remodelaciones en su 
Cuadro I.Análisis FODA de la Política Social

\begin{tabular}{|c|c|}
\hline FORTALEZAS & DEBILIDADES \\
\hline $\begin{array}{l}\text { Fondos Etiquetados para } \\
\text { fortalecimiento municipal y obras } \\
\text { públicas. }\end{array}$ & $\begin{array}{l}\text { Problemas en la implementación de } \\
\text { la política en el ámbito municipal }\end{array}$ \\
\hline $\begin{array}{l}\text { Beneficio para colonias en condiciones } \\
\text { de rezago social e índices de } \\
\text { marginidad. }\end{array}$ & $\begin{array}{l}\text { Dependencia de la capacidad } \\
\text { individual de los responsables } \\
\text { de su implementación }\end{array}$ \\
\hline $\begin{array}{l}\text { Construcción de nuevas obras o } \\
\text { remodelación de otras }\end{array}$ & $\begin{array}{l}\text { Falta de capacitación a servidores } \\
\text { públicos }\end{array}$ \\
\hline $\begin{array}{l}\text { Apoyo a varias colonias incluyendo la } \\
\text { del centro mejorando la imagen urbana }\end{array}$ & Falta de planeación a largo plazo \\
\hline $\begin{array}{l}\text { Estímulo para elevar el nivel de vida de } \\
\text { los ciudadanos }\end{array}$ & \\
\hline OPORTUNIDADES & AMENAZAS \\
\hline $\begin{array}{l}\text { Incrementar los beneficios a la sociedad } \\
\text { Solucionar las demandas presentes y } \\
\text { futuras en cuanto a obras públicas e } \\
\text { infraestructura. }\end{array}$ & $\begin{array}{l}\text { Recorte de presupuesto de los Fondos } \\
\text { etiquetados. } \\
\text { Desaparición de la Política Social del } \\
\text { programa federal. }\end{array}$ \\
\hline
\end{tabular}

Fuente: Elaboración propia con base en el Manual del Ramo 33,2003.

infraestructura, instalaciones y servicios. Los gastos ascienden actualmente a $\$ 124$ millones de pesos (Rangel, 2003).Asimismo, se concesionaron tres muelles internacionales, el primero, el Muelle Turístico Internacional, otorgado a la empresa Terminal Marítima del Sureste, S.A. de C.V., en 1996 por un periodo de 20 años, el monto de inversión asciende a 20 millones de dólares (Cardoso, 2004). La concesión del muelle turístico Puerta Maya, fue otorgada por 20 años al Grupo $\mathrm{H}$, que realizó una inversión de 30 millones de dólares en el desarrollo de toda la terminal y comenzó operaciones en enero de 1998. Actualmente, la administración está a cargo de la compañía Cozumel Cruise Terminal S.A. de C.V., parte del grupo Carnival Corporation (Uch, 2003). La tercera concesión fue la del muelle turístico Punta Langosta, Cozumel, S.A. de C.V., otorgada en 
Administración municipal y

políticas públicas para la

creación de infraestructura

Cuadro 2. Análisis FODA de la Política de privatización mediante concesiones

\begin{tabular}{|c|c|c|}
\hline 134 & FORTALEZAS & DEBILIDADES \\
\hline & $\begin{array}{l}\text { Construcción de muelles turísticos } \\
\text { internacionales, remodelación, } \\
\text { expansión y mejoramiento del } \\
\text { aeropuerto internacional. }\end{array}$ & $\begin{array}{l}\text { Falta de cultura turística. } \\
\text { Congestionamiento de tráfico en } \\
\text { las principales avenidas de la Isla }\end{array}$ \\
\hline & $\begin{array}{l}\text { Beneficio para la comunidad: } \\
\text { generación de empleo directo e } \\
\text { indirecto, incremento de arribos de } \\
\text { cruceros y pasajeros. }\end{array}$ & $\begin{array}{l}\text { Coordinación entre los empresarios } \\
\text { que ofrecen distintos servicios } \\
\text { turísticos }\end{array}$ \\
\hline & Derrama económica para la federación & $\begin{array}{l}\text { Falta de obras públicas e } \\
\text { infraestructura, calles, drenaje, etcétera }\end{array}$ \\
\hline & OPORTUNIDADES & AMENAZAS \\
\hline & $\begin{array}{l}\text { Realizar convenios de concesión hacia } \\
\text { otro tipo de servicios. } \\
\text { Modernización de otro tipo de obras: } \\
\text { agua potable, residuos sólidos, etc.. }\end{array}$ & $\begin{array}{l}\text { Sobrepasar la capacidad de pasajeros } \\
\text { que de acuerdo con la capacidad física } \\
\text { de la Isla se deben recibir. } \\
\text { Surgimiento de mayores problemas } \\
\text { socioculturales: pérdida de } \\
\text { constumbres, lenguaje, drogadicción, } \\
\text { alcoholismo, etc. } \\
\text { Incapacidad para eliminar los } \\
\text { residuos sólidos y líquidos } \\
\text { generados tanto por el turismo } \\
\text { como por la comunidad. }\end{array}$ \\
\hline
\end{tabular}

Fuente: Elaboración propia con base en las entrevistas realizadas, 2003 y 2004

1997 por un plazo de 25 años, las inversiones en este muelle son de alrededor I3 millones de dólares, y los gastos de operación anual son aproximadamente de 300000 dólares (Ríos, 2004). En el cuadro 2, se anotan las fortalezas, debilidades, amenazas y oportunidades de la política de privatización mediante concesiones. 


\section{Conclusiones}

Se observa la poca participación de ingresos captados por el municipio, destacándose que los generados por arrendamiento y concesiones representan altas cantidades y que a la par de la pretendida descentralización, la administración de los recursos sigue centralizada a cargo del gobierno federal, esto es una limitante para el desarrollo del municipio y por ende de la inversión en obras públicas e infraestructura.

Es indispensable que las autoridades comiencen a trabajar con base en la planeación estratégica, llevando a cabo un diagnóstico e identificando las amenazas, oportunidades, fortalezas y debilidades existentes, $y$ en esa medida plantear los objetivos claros y concretos a un largo plazo e insertarlos en los planes de trabajo municipal.

Uno de los problemas detectados es el incorrecto diseño o ejecución de la política social en el ámbito municipal, ya que de los recursos asignados, el promedio ejecutado fue de $70 \%$, es decir, el $30 \%$ restante fue reintegrado a la federación. Mientras que la política de privatización por concesiones ha resultado una buena opción para el mantenimiento de la infraestructura de acceso y brindar la calidad que demandan los visitantes.

En razón de lo anterior, las perspectivas para el municipio a corto plazo se consideran favorables, sin embargo, autoridades y prestadores de servicios deberán trabajar en conjunto y lograr consensos al margen de la capacidad física de la Isla, con la finalidad de atender a los turistas que visitan el destino en el marco de un desarrollo sustentable, a efecto de no dañar los recursos naturales existentes, como las condiciones de vida de la población local. Considerando que aún es tiempo de afrontar y darle solución a algunos problemas detectados tales como: el congestionamiento de tráfico en las principales avenidas en los días con mayor atraque de cruceros, falta de drenajes, dotación de agua potable, disposición final de residuos sólidos, tratamientos de aguas negras y transporte público, problemas que urge atender ya que cada día son más graves.

\section{Fuentes consultadas}

Aguilar Villanueva, Luis F. (1992). El estudio de las políticas públicas. Estudio introductorio. México: Porrúa, 28I p. 
Administración municipal y

políticas públicas para la

creación de infraestructura

Boullon, Roberto C. (1990). Municipios turísticos. México: Trillas.

Cortés Lozano, Benjamín (200I). El municipio información y gestión. México: Fundación Universitaria de Derecho Administrativo y Política, p. 10.

Garza Villarreal, Gustavo (1998). La gestión municipal en el área metropolitana de Monterrey, 1989-1994. México: Instituto de Investigaciones Sociales, UNAM, Porrúa.

Gobierno del Estado de Quintana Roo (2002). Programa Subregional de Desarrollo Urbano del corredor Cancún-Riviera Maya, (Región Caribe Norte), Quintana Roo, México: Gobierno del Estado de Quintana Roo.

(2003). Manual del Ramo 33.. Quintana Roo.

H.Ayuntamiento Municipal (200I) Dictamen de la cuenta Pública del H.Ayuntamiento del municipio de Cozumel, ejercicio fiscal 1996-2000.

-------- (1999). Ley Orgánica Municipal del estado de Quintana Roo LOMEQRO.

--------- (1999). Actas de Cabildo del municipio de Cozumel de enerodiciembre 1996-1998.

INEGI (Instituto Nacional de Estadística, Geografía e Informática) (2000). Cozumel, Estado de Quintana Roo, Cuaderno estadístico municipal, México: INEGI

Ruiz Caro, Ariela (2002). El proceso de privatización en el Perú durante el periodo 199I-2002. Chile: CEPAL, Ilpes.

ENTREVISTAS

Rangel, Cuahutémoc. Administrador general del Aeropuerto Internacional, Cozumel Quintana Roo, 14 abril 2003.

Uch Mezo, Alberto. Gerente de operaciones muelle turístico "Puerta Maya”. Cozumel Quintana Roo, 17 diciembre 2003.

Cardoso, Alberto. Gerente de operaciones del muelle turístico internacional, Cozumel Quintana Roo, I5 enero 2004.

Ríos Covían y Ruíz del Hoyo, José. Gerente general del muelle Turístico Punta Langosta, Cozumel Quintan Roo, 02 febrero 2004. 